\title{
Reduced excitability of the motor cortex in untreated patients with de novo idiopathic "grand mal" seizures
}

\author{
V Delvaux, G Alagona, P Gérard, V De Pasqua, P J Delwaide, A Maertens de Noordhout
}

\begin{abstract}
Objectives-Transcranial magnetic stimulation (TMS) was used to investigate motor cortex excitability, intracortical excitatory, and inhibitory pathways in 18 patients having experienced a first "grand mal" seizure within 48 hours of the electrophysiological test. All had normal brain MRI, and were free of any treatment, drug, or alcohol misuse. Results were compared with those of 35 age matched normal volunteers.

Methods-The following parameters of responses to TMS were measured: motor thresholds at rest and with voluntary contraction, amplitudes of responses, cortical silent periods, and responses to paired pulse stimulation with interstimulus intervals of 1 to $20 \mathrm{~ms}$.

Results-In patients, there were significantly increased motor thresholds with normal amplitudes of motor evoked potentials (MEPs), suggesting decreased cortical excitability. Cortical silent periods were not significantly different from those of normal subjects. Paired TMS with short interstimulus intervals (1-5 ms) induced normal inhibition of test MEPs, suggesting preserved function of GABAergic intracortical inhibitory interneurons. On the contrary, the subsequent period of MEP facilitation found in normal subjects (ISIs of 6-20 ms) was markedly reduced in patients. This suggests the existence of abnormally prolonged intracortical inhibition or deficient intracortical excitation. In nine patients retested 2 to 4 weeks after the initial seizure, these abnormalities persisted, although to a lesser extent.
\end{abstract}

Conclusion-The present findings together with abnormally high motor thresholds could represent protective mechanisms against the spread or recurrence of seizures.

(F Neurol Neurosurg Psychiatry 2001;71:772-776)

Keywords: epilepsy; cortical excitability; transcranial magnetic stimulation

Although the pathophysiological mechanisms of the epilepsies are obviously multiple, there seems to be marked differences between focal epilepsies, where there could be an imbalance between hyperexcitability of pyramidal cells and loss of activity of inhibitory interneurons, and non-convulsive absence epilepsy, in which thalamic GABAergic inhibitory neurons may have a crucial synchronising role. ${ }^{12}$ These different pathophysiological mechanisms are highlighted by the opposite effects of the administration of GABA agonists and antagonists in rats with either absence-like epilepsy or tonic audiogenic seizures. ${ }^{3-5}$ On the other hand, agents which block synaptic inhibition, such as penicillin or bicucullin, are known to induce epileptic discharges, ${ }^{6}$ again pointing to the functional importance of intracortical inhibitory structures. For these reasons, non-invasive measurements of cortical excitability and intracortical inhibitory function can be a useful approach to study the pathophysiology of human epilepsy. In this respect, transcranial magnetic stimulation (TMS) of the motor cortex seems promising. Indeed, the motor cortex is known to be a very epileptogenic structure and TMS allows measurements of several relevant parameters: motor cortex excitability, excitatory and inhibitory output to spinal motor neurons, and intracortical inhibition. Several authors have used TMS in epileptic patients, with conflicting results (for a review, see Ziemann et $\left.a l^{7}\right)$. Such discrepancies can be explained by several factors: heterogeneity of the populations studied and of the techniques used and particularly the fact that most patients were under different antiepileptic drug (AED) treatment regimens. Indeed, it has been shown that different AEDs can have opposite effects on responses to TMS in normal subjects. ${ }^{8-10}$ Moreover, alcohol or psychotropic drugs can also modify cortical excitability. ${ }^{11}{ }^{12}$

These reasons prompted us to choose a very selected population of patients to undertake the present study: patients with de novo idiopathic "grand mal" seizure without any history of drug or alcohol intake and receiving no AEDs.

\section{Patients and methods}

\section{PATIENTS}

With approval of the ethics committee of the University Hospital of Liege, we studied 18 patients (13 men, five women, aged 17-62, median age 28 years) who experienced a first "grand mal" seizure in the previous 48 hours without provocative factors. They all gave informed consent to the study. None of the patients had a history of neurological illness or trauma, and they were currently receiving no drug treatment. Six patients reported some degree of sleep deprivation before the initial seizure. None of them had a familial history of epilepsy. Patients with alcohol or illicit drug 
Table 1 Electroencephalographic characteristics of patients

\begin{tabular}{llll}
\hline Patient & Age & $\begin{array}{l}\text { Immediately } \\
\text { postictal EEG }\end{array}$ & $\begin{array}{l}\text { Interictal } \\
\text { EEG }\end{array}$ \\
\hline $1^{\star}$ & 17 & AT & SD \\
$2^{\star}$ & 25 & S & S \\
3 & 20 & SD & IW \\
4 & 18 & IW & N \\
5 & 45 & SD & IW \\
6 & 26 & AT & IW \\
7 & 39 & SD & SD \\
8 & 61 & SD & N \\
$9{ }^{\star}$ & 20 & SS & S \\
$10^{\star}$ & 30 & AT & AT \\
$11^{\star}$ & 32 & AT & IW \\
$12^{\star}$ & 38 & S & SD \\
$13^{\star}$ & 34 & SS & S \\
$14^{\star}$ & 33 & S & S \\
$15^{\star}$ & 40 & SS & SS \\
$16^{\star}$ & 22 & IW & S \\
$17^{\star}$ & 34 & SS & SS \\
$18^{\star}$ & 27 & IW & N \\
\hline
\end{tabular}

$\mathrm{SD}=$ Generalised slow dysrythmic; $\mathrm{IW}=$ intermittent slow waves; $\mathrm{S}=$ generalised spikes; SS=generalised multiple spikes; $\mathrm{AT}=$ generalised angular theta; $\mathrm{N}=$ normal.

*Patients tested twice.

intake or metabolic derangement were excluded, as well as those whose history suggested focal, secondarily generalised seizure. All patients had normal brain MRI. Most of them (12 of 18) experienced further "grand mal" seizures during a follow up period of up to 2 years. Several interictal and post-ictal EEGs were available for each patient. Characteristics of patients' EEGs are given in table 1.

The patients were compared with a group of 35 neurologically normal control volunteers (16 men, 19 women, aged 20-68, median age 26 years).

\section{METHODS}

All patients were studied within 48 hours of the first seizure. They were seated in a comfortable armchair in a quiet, semidarkened room, with eyes open. Transcranial magnetic stimulation (TMS) was applied through a focal $(2 \times 70 \mathrm{~mm})$ coil placed tangentially over the hand representation area of the left motor cortex and connected to a Bistim module (Magstim Ltd, UK) for paired stimuli. The handle was held posterior and downwards, perpendicular to the central sulcus. Electromyographic responses were recorded from the right first dorsal interosseous muscle (FDI) with a belly tendon montage with adhesive surface electrodes. Responses were filtered ( $3 \mathrm{~Hz}$ to $3 \mathrm{kHz}$ ), amplified with two different gains $(100 \mu \mathrm{V} / \mathrm{V}$ and $1 \mathrm{mV} / \mathrm{V}$ ), digitised (CED 1401 plus, Cambridge Electronic Design, UK), and stored for off line analysis (SigAvg program, Cambridge Electronic Design, UK). The level of background EMG activity was constantly monitored on an oscilloscope with auditory and visual feedback given to the subject.

The following parameters of FDI responses to TMS were measured: motor thresholds at rest and during isometric contraction, amplitude of the maximal responses to single TMS, duration of the EMG silent period induced by TMS, and responses to paired TMS.

Motor threshold (MT) was defined using the Bistim module as the minimum TMS intensity needed to evoke a motor evoked potential
(MEP) in the target muscle on $50 \%$ of 16 consecutive (signal gain $100 \mu \mathrm{V} / \mathrm{div}$ ) trials. Responses were recorded during full relaxation and under slight ( $20 \%$ maximum) isometric contraction of FDI as measured by quantitative preanalysis of the EMG background. ${ }^{12}$

The mean amplitude of the initial negative peak of five consecutive MEPs evoked by TMS at maximal stimulator output was measured and expressed as a percentage of the maximal response evoked in FDI by ulnar nerve stimulation (MEP max/Mmax ratio).

The mean duration of the EMG silent period (SP) elicited in FDI during maximal contraction was measured (10 consecutive trials). The stimulus intensity was set at $125 \%$ of MT threshold during isometric contraction and the $\mathrm{SP}$ was defined as the time from the end of the early response to the resumption of uninterrupted EMG activity at a display gain of 500 $\mu \mathrm{V} /$ div.

Paired TMS were delivered with interstimulus intervals (ISIs) ranging from 1 to $10 \mathrm{~ms}$ by $1 \mathrm{~ms}$ steps and from 10 to $20 \mathrm{~ms}$ by $5 \mathrm{~ms}$ steps. The experimental set up was similar to that described by others ${ }^{12}{ }^{13}$ except that the intensity of the conditioning stimulus was $95 \%$ of the motor threshold at rest (slightly above "active" or absolute motor threshold). This intensity of the conditioning stimulus was chosen to better express the period of facilitation after initial inhibition. It might, however, have the disadvantage of inducing a slight direct facilitation of spinal motor neurons by the descending volleys evoked by the conditioning stimulus so that the facilitation period might not be of purely intracortical origin. Intensity of the test TMS was $125 \%$ of the motor threshold at rest. Responses to paired TMS were recorded at rest. For each ISI, five conditioned responses were averaged and compared with five unconditioned MEPs. Test and conditioned responses were randomly intermixed for each block of four ISIs. Amplitudes of the first negative peak of conditioned responses were expressed as a percentage of the grand average of unconditioned MEPs. In two patients, paired TMS was repeated with a $90^{\circ}$ rotation of the coil, the handle being held lateral, to explore the effects of coil orientation on the modulation of paired TMS responses. In these patients, stimulus intensities were adjusted to produce responses of similar sizes as with the coil in the optimal orientation.

All results are expressed as means (SD) except for paired stimulation curves (mean (SEM)). Motor thresholds, SP duration, and maximal MEP amplitudes were compared using unpaired Student's $t$ test. Responses to paired TMS were compared with multivariate analysis of variance (MANOVA) and Tukey's post hoc analysis. In patients retested after 2 to 4 weeks, motor thresholds obtained at the two sessions were compared using Wilcoxon test. Differences between patients and normal subjects were considered significant for $\mathrm{p}$ values $<0.05$. 

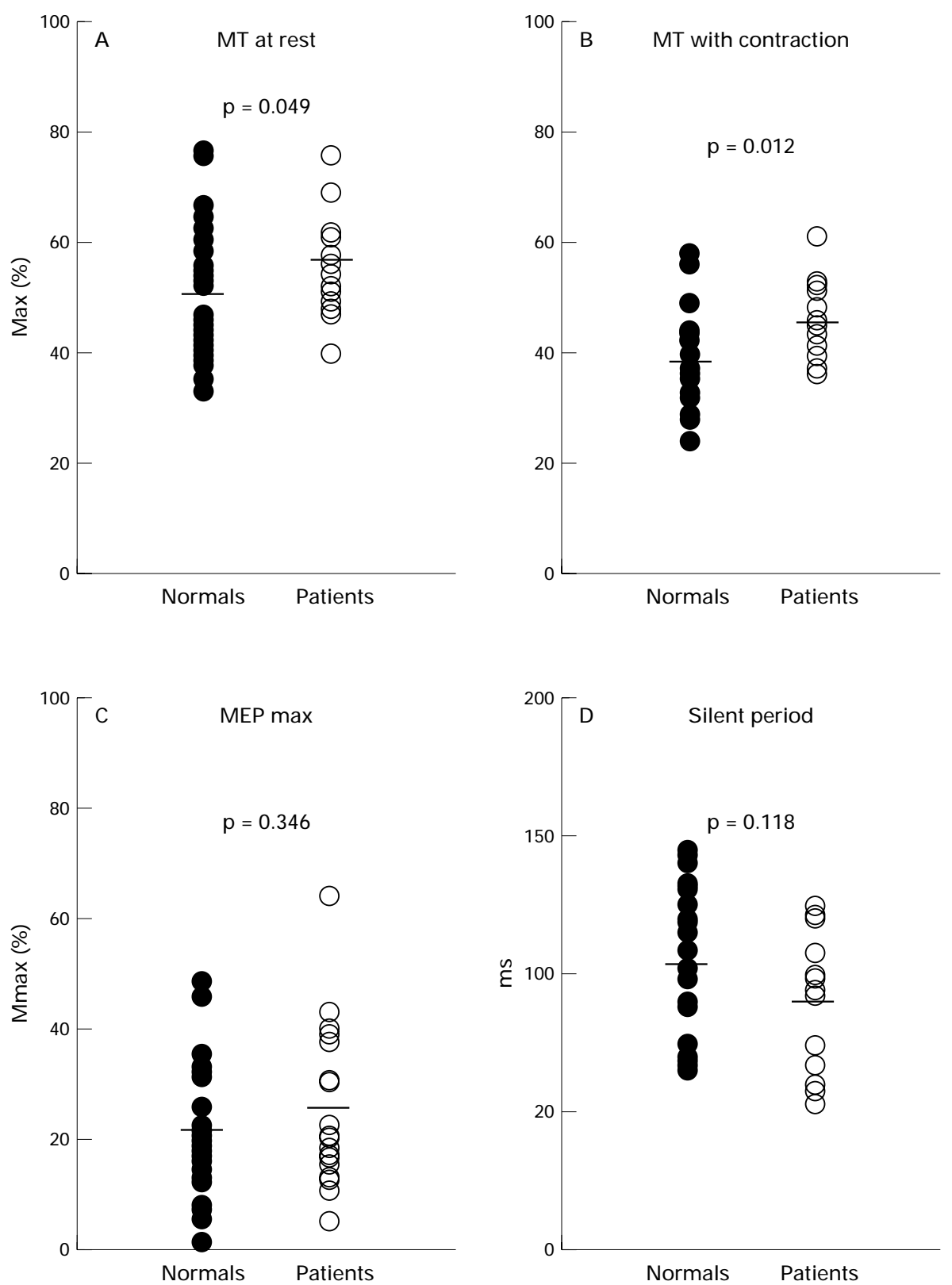

Figure 1 ( $A$ and $B)$ Individual values of motor thresholds (MTs) obtained during $(A)$ relaxation and $(B)$ voluntary contraction (20\% maximum) of the right FDI muscle of normal volunteers (left series) and patients (right series). The $M T s$ at rest and during contraction are significantly higher in patients than in normal subjects. (C and D) Individual values of (C) MEP amplitude (\% Mmax) and (D) of EMG silent period elicited by TMS (125\% MT) of normal volunteers (left series) and patients (right series). No significant differences were found for MEP amplitude or silent period duration between the two groups although the silent period tended to be shorter in patients.

\section{Results}

In normal subjects, FDI motor thresholds (MT) measured at rest and during isometric contraction were 51.0 (SD) 10.9 and 38.5 (SD) 8.5 (\% of maximal output). In epileptic patients, MTs were significantly higher, at rest as well as during contraction (57.0 (8.9) and 45.1 (SD 7.1); $\mathrm{p}=0.049$ and 0.012 , unpaired $t$ test, fig 1 ). In nine patients retested 2 to 4 weeks after the initial seizure, resting MTs were significantly lower than at first test (54.9 (SD 6.1) $v 59.2$ (SD 6.9), $\mathrm{p}=0.04$, Wilcoxon) whereas active MTs were also reduced (41.3 (SD 3.7) v 44.6 (SD 6.4) but not significantly ( $p=0.15$, Wilcoxon). Resting and active MTs measured at the second session were not significantly different from those of normal subjects $(\mathrm{p}=0.31$ and 0.34 , unpaired $t$ test $)$.

Maximal amplitude of the initial MEP negative peak was not significantly different between normal subjects (21.8 (SD 11.8)\% of Mmax) and patients (25.8 (SD 15.6)\% of Mmax, $\mathrm{p}=0.35$, unpaired $t$ test, fig 1 ).

The mean duration of the EMG silent period elicited in the FDI by cortical stimuli (intensity $125 \%$ of MT) was 103.7 (SD 25) ms in normal subjects and 89.3 (SD 24.5) $\mathrm{ms}$ in epileptic patients ( $\mathrm{p}=0.12$, unpaired $t$ test, fig 1$)$. The SP was not measured in three patients because of technical problems. 


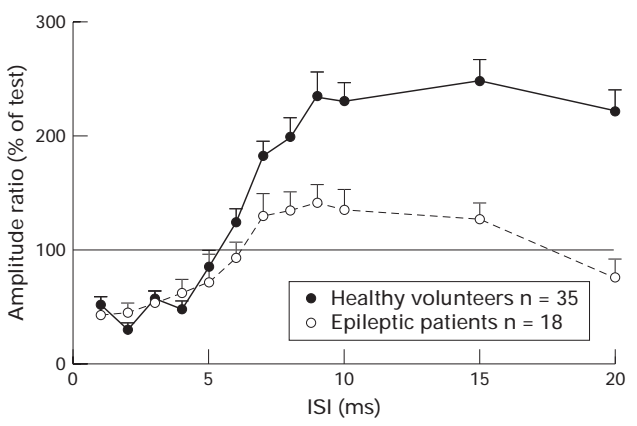

Figure 2 Average responses to paired TMS (\% of unconditioned responses) obtained in 35 healthy volunteers and 18 untreated epileptic patients tested within 48 hours of seizure. Values are expressed as mean values (SEM). The facilitatory period (ISIs from 6-20 ms) was significantly less prominent in patients than in normal subjects (see text for details).

Motor evoked potentials to paired TMS at rest were similarly inhibited with short interstimulus intervals (ISI from 1 to $5 \mathrm{~ms}$ ) in normal subjects and in epileptic patients (fig 2). In normal subjects, this early inhibition was followed by a clear cut facilitation of test responses with longer ISIs (6 to $20 \mathrm{~ms}$ ). In patients, this phase of facilitation was clearly reduced, with significant differences $(p=0.00014$, MANOVA and Tukey's post hoc analysis). In nine patients re-examined 15-30 days after the seizure, the "late" facilitation tended to reappear (fig 3) but remained less marked than in normal subjects $(p=0.017$, MANOVA and Tukey's post hoc analysis). In two patients showing absent facilitation of conditioned TMS for ISIs from 6 to $20 \mathrm{~ms}$, a lateral $90^{\circ}$ rotation of the coil handle lateral did not modify the excitability curves, provided that the stimulus intensities were adjusted in function of MT values obtained with the new coil orientation.

\section{Discussion}

Patients examined in this study are certainly not representative of a general population of epileptic patients. When examined for the first time, they could not be considered as epileptic on the basis of a single fit but most of them experienced further "grand mal" seizures during follow up. This group was chosen because of its homogeneity and the absence of any drug treatment.

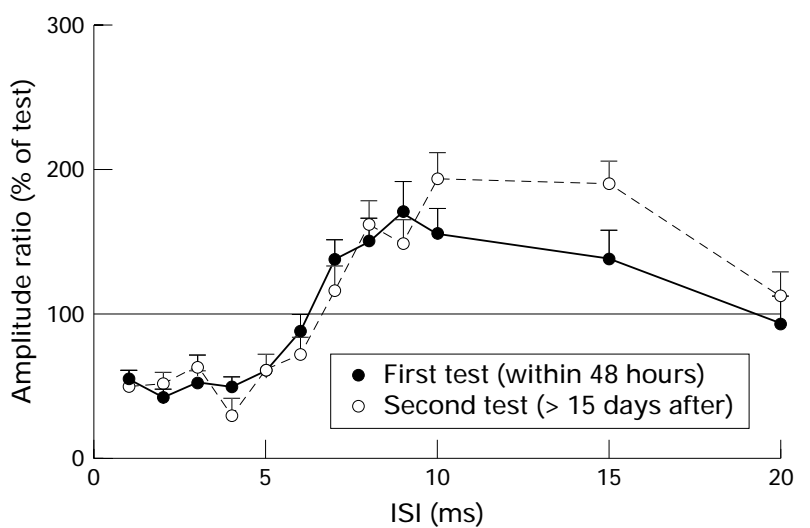

Figure 3 Average responses to paired TMS (\% of unconditioned responses) obtained in nine epileptic patients within 48 hours from seizure (solid line) and 2 to 4 weeks later (dashed line). Although facilitation found for ISIs of 6-20 ms tended to increase at the second testing, it remained significantly less marked than in normal subjects (see text).
Compared with normal subjects, patients showed abnormally high MTs contrasting with normal maximal MEP amplitudes, slightly but not significantly shorter cortical silent periods, and lack of MEP facilitation on paired TMS with ISIs from 6 to $20 \mathrm{~ms}$, whereas the early period of inhibition was preserved. High MTs were found by several authors in various subtypes of epilepsy ${ }^{14}$ but most patients studied were under AEDs, which can indeed modify motor cortex excitability. ${ }^{16}$ There have been few studies of untreated patients. Reutens et $a I^{17}$ found reduced motor thresholds in untreated patients with idiopathic generalised epilepsy whereas Gianelli et a ${ }^{18}$ found the opposite in similar patients. The discrepancy between these results is unclear as methods and patients were similar. Modifications of MT might be a dynamic phenomenon with variations locked to the timing of seizures. Indeed, MTs were higher in nine patients studied within 24 hours of the first seizure than when retested 2 to 4 weeks later. As maximal MEP amplitudes were similar in patients and in normal subjects, increased MT values found in epileptic patients do not reflect deficient motor cortex excitatory output. Also, there are no arguments in favour of reduced excitability of spinal motor neurons in untreated epileptic patients, so that MT changes are likely of cortical origin. The increase in MT could simply reflect dysfunction of the motor cortex after the seizure but it could also be a protective mechanism against spread or recurrence of seizures.

This hypothesis seems to be strengthened by the results of paired TMS. Although the early (ISIs from 1 to $5 \mathrm{~ms}$ ) inhibition was similar in both groups, the subsequent (ISIs from 6 to 20 ms) facilitation found in healthy subjects was markedly reduced in epileptic patients. The early (ISIs of 1-5 ms) period of inhibition is thought to result from the activation by conditioning TMS of inhibitory, probably GABAergic, intracortical interneurons, ${ }^{13}{ }^{19}$ the function of which seems preserved in the population studied. Recently, Brodtmann et aI, ${ }^{20}$ using long ISIs (200 to $300 \mathrm{~ms}$ ) correlated with the mean interspike interval on EEG, found a period of increased facilitation in untreated epileptic patients. Such a late facilitation is unlikely to result from activity of intracortical excitatory interneurons directly connected to pyramidal tract neurons, which are thought to be responsible for the facilitation induced for short ISIs (6-20 ms). ${ }^{13}{ }^{19}$ With the present experimental set up (conditioning stimulus slightly above active MT), a small part of the facilitation of the test stimulus can also result from increased excitability of spinal motor neurons by the weak descending volleys evoked by the conditioning. This effect must be short lasting (3-5 ms, depending on the number of descending volleys evoked by the conditioning), so that most of the facilitation found must have originated at a cortical level. The reason why we used slightly supraliminal conditioning pulses was to show the facilitation better than with the conventional (slightly subthreshold) method.

In our patients, the lack of MEP facilitation with paired TMS suggests the existence of 
either deficient intracortical facilitation or abnormally prolonged intracortical inhibition. In two patients, the curves were not modified when the coil handle was rotated by $90^{\circ}$. This suggests that diffuse intracortical pathways were responsible for that absent facilitation rather than specifically oriented ones, favouring the hypothesis of abnormally prolonged inhibition rather than deficient facilitation. Indeed, it has been shown that the facilitation on paired TMS is maximal when the coil handle is held perpendicular to the central sulcus, with probable activation of specifically oriented (posteroanterior) cortical interneurons. Another explanation could be that with the intensity used for the conditioning pulse (between active and resting MT), corticospinal volleys induced in the pyramidal tract of patients were smaller than in normal subjects, thus less prone to facilitate the test response. We think that this is unlikely as the intensities used were adjusted in each subject as a function of individual MTs and maximal amplitudes of MEPs were similar in patients and normal subjects.

To date, paired TMS studies were conducted in partial epilepsy or cortical myoclonus with findings of decreased intracortical inhibition. ${ }^{21}{ }^{22}$ However, all patients from these studies were under AEDs so that definitive conclusions cannot be drawn.

Contrary to the findings of Gianelli et $a l,{ }^{18}$ we did not find reduced MEP amplitudes in epileptic patients. However, their measurements were taken during the slow wave of the spike and wave EEG complex - that is, during hyperpolarisation of pyramidal cells.

Cortical silent periods were slightly but not significantly shorter in our patients than in healthy volunteers. Classen et $a l^{23}$ reported prolonged SPs in partial epilepsy on the side of the focus but again their patients were under AEDs, which can modify SPs.

\section{Conclusion}

The present study conducted on a very selected population of patients indicates that cortical excitability seems to be reduced after a seizure in idiopathic "grand mal", epilepsy without any treatment and that intracortical GABAergic inhibition might be prolonged. These abnormalities faded after 2 to 4 weeks, but results remained significantly different from those of normal subjects. We interpret these findings as a putative protective mechanism against spread or recurrence of seizures. It remains to be determined whether the same type of abnormalities are found in partial epilepsy or in non-convulsive absence seizures and the effects of antiepileptic treatments. The present findings could also account for the fact that seizures can seldom be induced in epileptic patients by repetitive TMS, which can be epileptogenic in normal subjects.

This study was supported by a grant from the Fondation Leon Fredéricq for Medical Research at the University of Liege. Valérie Delvaux was funded as clinical research fellow by the Medical Research Fund of the Centre Hospitalier Universitaire of Liège.

1 Depaulis A, Deransart C, Vergnes M, et al. Mécanismes GABAergiques dans les épilepsies généralisées: Ia dimenGABAergiques dans les epilepsies generalisees: Ia dimen-
sion neuroanatomique. Rev Neurol (Paris) 1997;153(suppl sion neur $8-13$.

2 Bernard C, Hirsch J, Ben An Y. Récepteurs excitateurs glutamatergiques et épilepsie du lobe temporal. Rev Neurol (Paris) 1997;153(suppl 1):14-24.

3 Bradford HF. Glutamate, GABA and epilepsy. Prog Neurobiol 1995;47:477-511.

4 Vergnes M, Marescaux C, Micheleti G, et al. Enhancement of spike and wave discharges by GABA mimetic drugs in rats with spontaneous petit-mal-like epilepsy. Neurosci Lett 1984;44:912-14.

5 Vergnes M, Boehrer A, Simler S, et al. Opposite effects of GABAB receptor antagonists on absences and convulsive seizures. Eur f Pharmacol 1997;332:245-55.

6 Jefferys JGR. The pathophysiology of epilepsies. In: Laidlaw J, Richens A, Chadwick D, eds. A textbook of epilepsy. Edinburgh: Churchill Livingstone, 1993:241-71.

7 Ziemann U, Steinhoff BJ, Tergau F, et al. Transcranial magnetic stimulation: its current role in epilepsy research. Epilepsy Res 1998;30:11-30.

8 Mavroudakis N, Caroyer JM, Brunko E, et al. Effects of diphenylhydantoin on motor potentials evoked with magnetic stimulation. Electroencephalogr Clin Neurophysiol 1994;93:428-33.

9 Mavroudakis N, Caroyer JM, Brunko E, et al. Effects of vigabatrin on motor potentials with magnetic stimulation. Electroencephalogr Clin Neurophysiol 1997;105:124-7.

10 Ziemann U, Lönnecker S, Steinhoff BJ, et al. Effects of antiepileptic drugs on motor cortex excitability in humans: a transcranial magnetic stimulation study. Ann Neurol 1996; 40:367-78.

11 Ziemann U, Lönnecker S, Paulus W. Inhibition of human motor cortex by ethanol. A transcranial magnetic stimulation study. Brain 1995;118:1437-46.

12 Triggs WJ, Cros D, MacDonell RAL, et al. Cortical and spinal motor excitability during the transcranial magnetic nal motor excitability during the transcranial magnetic
stimulation silent period in humans. Brain Res 1993;628: 39-48.

13 Kujirai T, Caramia MD, Rothwell JC, et al. Cortico-cortical inhibition in human motor cortex. $\mathcal{f}$ Physiol (Lond) 1993;471:501-19.

14 Hufnagel A, Elger CE, Marx W, et al. Magnetic motorevoked potentials in epilepsy: effects of the disease and of anticonvulsant medication. Ann Neurol 1990;28:680-6.

15 Schüler P, Claus D, Stefan H. Hyperventilation and transcranial magnetic stimulation: two methods of activation of epileptiform EEG activity in comparison. F CIin Neurophysiol 1993;10:111-15.

16 Ziemann U, Lönnecker 5, Steinhoff BJ, et al. The effect of lorazepam on the motor cortex excitability in man. Exp Brain Res 1996;109:127-35.

17 Reutens DC, Berkovic SF, Macdonneil RA, et al. Magnetic stimulation of the brain in generalized epilepsy: reversal of stimulation of the brain in generalized epilepsy: reversal of 1993:34:351-5.

18 Gianelli M, Cantello R, Civardi C, et al. Idiopathic generalized epilepsy: magnetic stimulation of the motor cortex time-locked and unlocked to $3 \mathrm{~Hz}$ spike-and-wave discharges. Epilepsia 1994;35:53-60

19 Ziemann U, Rothwell JC, Ridding MC. Interaction between intracortical inhibition and facilitation in human motor cortex. F Physiol (Lond) 1996;496:873-81.

20 Brodtmann A, Macdonell RAL, Gilligan AK, et al. Cortical excitability and recovery curve analysis in generalized epilepsy. Neurology 1999;53:1347-9.

21 Caramia MD, Gigli G, Iani C, et al. Distinguishing forms of generalized epilepsy using magnetic brain stimulation. Electroencephalogr Clin Neurophysiol 1996;98:14-19.

22 Brown P, Ridding MC, Werhahn KJ, et al. Abnormalities of the balance between inhibition and excitation in the motor cortex of patients with cortical myoclonus. Brain 1996;119: 309-17.

23 Classen J, Witte OW, Schlaug G, et al. Epileptic seizures triggered directly by focal transcranial magnetic stimulation. Electroencephalogr Clin Neurophysiol 1995;94:19-25. 